\title{
LA ADMINISTRACIÓN Y LOS AUDITORES DE PROCESAMIENTO AUTOMÁTICO DE DATOS (PAD) EN EL ENTORNO DE LAS EMPRESAS
}

\author{
MANAGEMENT AND AUDITORS OF AUTOMATIC DATA \\ PROCESSING (PAD) IN THE BUSINESS ENVIRONMENT
}

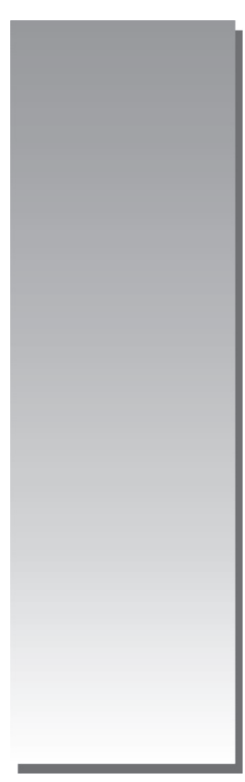

Félix Armando Rivera León*

felix_arl@hotmail.com

[RECEPCIÓN: MARZO DE 2015 / CONFORMIDAD: ABRIL DE 2015]

\section{RESUMEN}

Con el fin de establecer metas realistas y llevar a cabo sus funciones eficazmente, los auditores de procesamiento automático de datos (PAD) deben saber qué esperan sus empresas de ellos, además de poseer una comprensión clara de los objetivos de la administración. Este fascículo expresa estas cuestiones y describe algunas responsabilidades que deben existir entre el auditor de PAD y la administración en las empresas.

Palabras clave: Auditores, administración, entorno empresarial.

\section{ABSTRACT}

In order to set realistic goals and carry out its functions effectively, the auditors of automatic data processing (EDP), should know what they expect their companies. Possess a clear understanding of the objectives of the administration. This booklet expresses these issues and describes some responsibilities that must exist between the EDP auditor and management in enterprises.

Keywords: Auditors, management, business environment.

\footnotetext{
Doctor en Ciencias Contables y Empresariales (UNMSM); magíster en Administración con mención en Gestión Empresarial; contador público colegiado con especialidad en Auditoría (ESAN); con 36 años de experiencia profesional. Profesor principal de la Facultad de Ciencias Administrativas (UNMSM). Ha desempeñado diversos cargos administrativos en la facultad y en diferentes entidades del sector público y privado.
} 


\section{LA IMPORTANCIA DEL CONTROL INTERNO \\ (Eduardo J. Mencilla Perez, 2001, Cap. 6)}

Uno de los procesos más importantes de la auditoría durante las últimas décadas es la creciente comprensión, tanto de la administración como de los auditores, de la naturaleza complementaria existente en sus responsabilidades. Unido al deseo común del logro de la eficiencia y estados financieros dignos de confianza, esto ha fomentado un alto grado de colaboración entre ellos.

Por ello, considerar la naturaleza y características del control interno al delinear las respectivas esferas de interés y responsabilidades de la administración y los auditores, permite definir un proceso diseñado para proporcionar seguridad razonable mirando el cumplimiento de objetivos como:

- Efectividad y eficiencia de las operaciones.

- Confiabilidad de la información financiera.

- Cumplimiento de las leyes y regulaciones aplicables.

(Objetivos planteados por Samuel Mantilla - 1994)

\section{EL AUDITOR Y EL FRAUDE}

Son muchas las exposiciones y literatura que se ha desplegado donde no se llega a definir con claridad la responsabilidad del auditor en todas las áreas posibles de error, negligencia, fraudes, etcétera, en las prácticas de una auditoría.

Con el empleo diligente de las normas y procedimientos de auditoría, el auditor puede llegar a detectar modalidades de fraude o estafas, lo cual se refuerza si el auditor en su examen está atento a la posibilidad de que hayan ocurrido o se estén realizando actos ilegales que puedan tener efecto significativo en los estados financieros. (Normas Generales de los Sistemas Administrativos - 1979)

Debe quedar claro que no es objetivo de la auditoría la detección de errores o irregularidades, pero ello no significa que el auditor no esté alerta a la posible presentación de este tipo de situaciones en el curso normal de su tarea.

\section{OBLIGACIONES DEL AUDITOR}

Debe realizar sus prácticas de auditoría utilizando toda su capacidad, inteligencia y criterio para determinar el alcance, las estrategias y técnicas que habrá que emplear en una auditoría, cómo evaluar los resultados y presentar los informes correspondientes.

Por lo tanto, debe cumplir con:

- Poseer un entrenamiento y capacidad profesional como auditores.

- Poseer, en todos los asuntos relacionados con el examen, independencia de criterio.

- Esmero profesional en el desarrollo del examen.

- Poseer naturaleza confidencial del trabajo.

- Deberá eximirse de ejercer cualquier labor relacionada con la administración, aspectos financieros, en las entidades sujetas a su evaluación.

- Cumplir con normas o criterios que se le señalen.

El auditor no debe olvidar que la fortaleza de su función y proyección personal está sujeta en la medida en que afronte su compromiso con respeto y en apego a normas profesionales como:

- Objetividad.

- Responsabilidad.

- Integridad.

- Confidencialidad.

- Equilibrio.

- Honestidad.

- Institucionalidad.

- Criterio.

- Iniciativa.

- Imparcialidad.

- Creatividad.

(Resumen obtenido de Enrique Benjamin Franklin F - 2002 pgs. 19 al 37)

\section{EL AUDITOR DE PROCESAMIENTO DE DATOS (PAD)}

Frente a los sistemas de información computarizada, a la administración -incluidos los auditores- les preocupa la eficiencia de los procesos, la exactitud de los datos grabados y la adecuación de los procedimientos de control. El ordenador participa en todos estos 
aspectos. Por consiguiente, el auditor deberá adquirir los conocimientos necesarios para llevar a cabo estudios de sistemas y aplicaciones mecanizados y verificar datos que estén procesando.

La actividad de auditoría puede ser de muchos tipos, niveles de información y enfoques. Las auditorías pueden ser internas o externas a la empresa que se vaya evaluar. Los auditores pueden informar a niveles de finanzas, de dirección o al nivel externo independiente.

El auditor de procesamiento automático de datos (PAD), que es parte del equipo de auditoría, le preocupará básicamente el control interno, el control y la fiabilidad financiera, como la seguridad de los activos de la empresa. Muchos tendrán responsabilidades en las que se incluyan análisis de los sistemas y de los procesos empresariales. Algunos se interesarán en la eficacia, eficiencia, continuidad de los sistemas y en la exactitud de los casos que se presenten a la jefatura; otros auditores analizarán si el equipo informático y los sistemas de software son técnicamente adecuados y eficientes. Sería imposible encontrar un proceso específico de evaluación en auditoría para el auditor informático.

Por otro lado, el entorno en que se realizan los trabajos del auditor varía enormemente de un lugar a otro. Algunos sistemas son pequeños y forman parte de la organización administrativa; otros pueden ser grandes sistemas financieros independientes en batch; otros son mezcla de los anteriores; otros poseen terminales en teleproceso en departamentos usuarios; por último, otros son unas redes de sistemas combinados. Los enfoques de trabajo son variados según las aplicaciones de evaluación a realizar, aunque los objetivos de esta pudieran ser similares en circunstancias distintas.

La relación que debe complementarse entre los auditores y el auditor de proceso de datos (PAD) debe adquirir un conocimiento general del sistema informático, al mismo nivel del conocimiento conocido de los sistemas contables de las empresas. Reconociendo los puntos fuertes y débiles del funcionamiento del sistema informático y en aquellos que pudiera darse el caso de que los procedimientos fallen, los auditores informáticos deben tener una visión clara de cuál es su papel frente a la jefatura del equipo de auditoría y cada uno comprenderá la posición del otro.

Se espera que el auditor PAD tome las siguientes medidas: (consideraciones tomadas por Rodrigo Estupiñan Gaitan - 2006)

- Pensar siempre en el riesgo del auditor.

- El auditor no solo debe reconocer problemas, sino también convencer y alentar a la administración a tomar acciones correctivas oportunas.

- Evaluar los controles dentro de sistemas nuevos antes de que sean operacionales.

- Debe usar técnicas avanzadas para realizar procedimientos de auditoría (técnicas de análisis operacionales y matemáticos).

- Debe trabajar con el auditor que no es informático para ayudarlo a entenderse con sistemas automatizados.

- Debe proveer el puente interpretativo, el lenguaje técnico del departamento de procesamiento de datos y el lenguaje administrativo, para informar, promover el control, mejorar la comprensión de la auditoría.

\section{RESPONSABILIDAD DE LA ADMINISTRACIÓN}

(W. Thomas Porter Jr. 1980))

Las empresas, en su contexto administrativo, si quieren tener un futuro, no pueden ni deben basarse en formas y métodos que pertenecen al pasado, porque consecuentemente correrán graves riesgos al tratar nuevas actividades usando métodos, herramientas, planteamientos y normas anticuados, muchos de los cuales necesitarán ser sustituidos por otros más actualizados, o quienes dirigen no tengan el acierto de entenderlos o la capacidad de interpretarlos para accionar apropiadamente ante ellos.

Una función eficaz de auditoría informática necesitará el apoyo de la administración que comprometa a toda la empresa. Sin los recursos necesarios y el apoyo total de la empresa, el auditor no puede ser responsable de su cumplimiento personal o norma alguna de competencia.

El proceso de evaluación no se completa mientras el auditor no esté satisfecho con la pertinencia de los resultados, producto de sus indagaciones. A la demanda a la administración 
para que responda por los resultados de la auditoría, la empresa contribuirá a la eficacia de la función y aumentará la probabilidad de que reciban la atención conveniente.

\section{RECOMENDACIÓN}

Los auditores del PAD, al tomar plena conciencia del riesgo, pueden aportar más a la empresa, deben ser observadores experimentados, usando sus aptitudes para determinar los puntos fuertes y débiles de la empresa, sus oportunidades y amenazas, problemas, la calidad de sus productos, la dignidad, el interés de sus empleados y las deficiencias de sus operaciones. Cuando un auditor conoce íntegramente a la empresa que evalúa, el conocimiento del riesgo se hace más significativo, otorgando mayor eficiencia a los resultados de la auditoría que realiza, así como de la comunicación efectiva con la administración.

\section{LITERATURA CITADA}

Contraloría General de la República. Normas Generales de los Sistemas Administrativos, Sistema de Racionalización, Resolución Jefatural No $182-79-I N A P / P N R$

William P. Leonard. 1980. Auditoría Administrativa. Editorial Diana. México.

Franklin F. Enrique Benjamín. 2001. Auditoría Administrativa. McGraw-Hill Interamericana. México.

Espiñan Gaitán Rodrigo. 2006. Control interno y fraudes. Ecoe Ediciones, Bogotá.

Mantilla B. Samuel Alberto. 2002. Control Interno, estructura conceptual integrada. Committee of Sponsoring Organizations of the Treadway Commission (COSO),Bogotá.

W. Thomas Porter Jr. y Jhon C. Burton.1980. Auditoría: Análisis Conceptual. Editorial Diana. México. pgs. 207-208. 